\title{
Efficient extraction of canonical spatial relationships using a recursive enumeration of k-subsets
}

\author{
Georg Hinselmann*, Nikolas Fechner, A Jahn, Andreas Zell \\ From 5th German Conference on Cheminformatics: 23. CIC-Workshop \\ Goslar, Germany. 8-10 November 2009
}

The spatial arrangement of a chemical compound plays an important role regarding the related properties or activities. A straightforward approach to encode the geometry is to enumerate pairwise spatial relationships between $k$ substructures, like functional groups or subgraphs. This leads to a combinatorial explosion with the number of features of interest and redundant information. The goal of this work is to compute all possible $k$-subsets of spatial points and to extract a single canonical descriptor for each subset in sub-polynomial computation time. More precisely, the problem is to reduce the complexity of $n_{k}=n \cdot(n-1) \ldots(n-k)$ possible relationships (patterns or descriptors) for $n$ features and $k$-point relationships.

We propose a two-step algorithm to solve this problem. A modified algorithm for the computation of the binomial coefficient computes the $k$-subsets [1] containing the possible combinations of the $n$ relevant features. If a $k$-subset is completed in the inner recursion, the algorithm computes a canonical representation for it. By defining a natural order by means of the geometrical center of gravity of the $k$ points, we extract $k$ patterns that describe the distance to the center of gravity and type of the spatial feature $k \in F$. Then, the algorithm returns a unique identifier for the lexicographically sorted array of patterns. If applicable $\left(f_{\pi_{0}}<f_{\pi_{1}}<\ldots f_{\pi_{k}}\right)$, an additional identifier is added which has the form $f_{\pi_{o}} \stackrel{d_{\pi_{0} \pi_{1}}}{\rightarrow} f_{\pi_{1}} \stackrel{\ldots}{\rightarrow} \ldots f_{\pi_{k-1}} \stackrel{d_{\pi_{k-1} \pi_{k}}}{\rightarrow} f_{\pi_{k}}$, where $d_{i j}$ denotes the geometrical distance between features $i$, $j$. Else $\left(f_{\pi_{o}} \leq f_{\pi_{1}} \leq \ldots f_{\pi_{k}}\right)$, this step is omitted. Therefore, this approach also considers stereochemistry. Finally, one

University of Tübingen, Sand 1, 72076 Tübingen, Germany feature is returned for each $k$-subset resulting in a set of $C(n, k)$ patterns describing the structure.

The main result is that the number of features is reduced from $n_{k}$ to $C(n, k)$, which equals the binomial coefficient. This procedure is useful in combination with similarity approaches that use spatial relationships, like pharmacophore searches, fingerprints, or graph kernels. We experimentally validated the algorithm on numerous QSAR benchmark sets in combination with the pharmacophore kernel [2].

Published: 4 May 2010

\section{References}

1. Rolfe T: SIGCSE BUll 2001, 33(3):35-36.

2. Mahé P, Ralaivola L, Stoven V, Vert J-P: J Chem Inf Mod 2006, 46(5):2003-2014

\section{doi:10.1186/1758-2946-2-S1-P36}

Cite this article as: Hinselmann et al: Efficient extraction of canonical spatial relationships using a recursive enumeration of k-subsets. Journal of Cheminformatics 2010 2(Suppl 1):P36.

\section{Publish with ChemistryCentral and every scientist can read your work free of charge \\ "Open access provides opportunities to our colleagues in other parts of the globe, by allowing anyone to view the content free of charge." W. Jeffery Hurst, The Hershey Company. \\ - available free of charge to the entire scientific community \\ - peer reviewed and published immediately upon acceptance \\ - cited in PubMed and archived on PubMed Central \\ - yours - you keep the copyright \\ Submit your manuscript here: \\ http://www.chemistrycentral.com/manuscript/

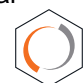 Chemistry Central}

